\title{
Application of thermal methods for characterization of steel welded joints
}

\author{
U. Galietti, D. Palumbo
}

Politecnico di Bari, Dipartimento di Ingegneria Meccanica e Gestionale, Viale Japigia 182 Bari, Italy

\begin{abstract}
Despite the large number of proposals in the field of fatigue prediction of welded joints, a globally accepted and unified theory, which applies easily to any load condition, does not exist. Real life components, indeed, differ in geometry and/or type of load from the structural design for which they are regarded by Standards, so that a lot of precautionary safety factors are used that lead to an underestimation of the actual fatigue life of joints. Infrared thermography has a great potential in this field, both from structural and thermomechanical points of view. It enables a full field stress analysis with a sufficient spatial resolution so that the complexity of the stress state at the weld toe and its time evolution are taken into account, emphasizing anomalies that may predict structural failure. A new methods for evaluation fatigue limit damage is presented in this paper and in particular interesting results derived from analysis of the evolution of thermoelastic signal phase. Variations in the value of signal phase indicate a not elastic behaviour and plastic dissipation in the material.
\end{abstract}

\section{Introduction}

Despite the large number of proposals in the field of fatigue prediction of welded joints, a unified theory and global validity, which applies easily to any load condition, does not exist. The design components in fact, differ in geometry and/or type of load from the structural details for which are regarded by rules so recourse usually a lot of precautionary safety factors that lead to an underestimation of the actual fatigue life of the joint.

Consolidated procedure were applied to determine the fatigue limit of materials from the analysis of the evolution of temperature during increasing loading [1-3].

Classical methods for evaluating the fatigue limit require very time consuming tests, so accelerated methods for analyzing the fatigue behaviour of both materials and welded joints are of great interest. There are already in literature $[2,3]$ thermal methods that allow the determination of fatigue limit and even the entire Wöhler curve. Anyway these methods are available only for base material testing of standard specimens and show many problems for materials different from steel. In particular, traditional methods require the determination of heat sources and therefore of plasticised area. 
In literature very few works have tried to use accelerated thermographic methods for studying the fatigue behaviour of components and welded joints.

In this work the feasibility of the use of well established techniques for base materials to evaluate the fatigue limit also for welded joints will be verified and, moreover, a new method based on thermoelastic data $[4,5]$ for assessment the fatigue limit more easily, quickly and reliably, will be presented.

Furthermore, the possibility to use the phase information of the TSA technique to determine the plasticised area, will be also demonstrated.

A fatigue test on three welded joints specimens manufactured from steel $\mathrm{Fe} 37$ are conducted.

The entire test was monitored with infrared thermocamera, providing, at regular intervals of time, to acquire the registration of thermoelastic images and thermographic sequences.

Interesting results derived from analysis of the evolution of thermoelastic signal phase: variations in the value of signal phase indicate an not elastic behaviour and plastic dissipation in the material [4, 5]. The phase changes are due to high gradients of stress that may undermine the adiabatic conditions and to the plastic behaviour of the crack tip.

A new method is also presented based on the analysis of the evolution of the phase change over time during the same loading stepped procedure that is much simpler and with an higher signal to noise ratio and then is entitled to give good results also with difficult materials such as aluminium alloys.

\section{Theory}

Metallic materials during a cyclic loading fatigue test show a characteristic heating linked directly to the damage of material. Various authors have show that the damage provides a likely indication of the defect position. Recent works have show that the temperature is not directly related to fatigue damage. Temperature, in fact, depends of the amplitude stress, of the test frequency, of the specimen geometry and environmental conditions that affect the heat exchange conditions between the specimen and the environment.

A thermomechanical model proposed by Chrysocoos et al to steel welded joints of rectangular section subjected to fatigue test, will be used in this work.

The Thermodynamics of Irreversible Process concept was the thermomechanical framework used to interpret the experimental results, this concept is based on the local state axiom and consequently characterizes the equilibrium state of each volume material element through a set of $\mathrm{N}$ state variables.

The selected variables are the absolute temperature $\mathrm{T}$, the linearized strain tensor e and N-2 scalar components $\alpha_{1}, \alpha_{2}, \ldots, \alpha_{\mathrm{N}-2}$ of the vector $\alpha$ gathering the internal variables. These latter describe the macroscopic effects of complex and often coupled microstructural phenomena.

Using the generalized standard materials formalism it is possible to sum-up the material thermomechanical behaviour by means of a thermodynamic potential and a dissipation potential. By construction, the thermodynamic potential is the specific Helmholtz free energy $\Psi\left(T, \varepsilon, \alpha_{i}\right)$ and the dissipation potential is a function of the state variable fluxes [2].

In the follow model we consider $\alpha_{0}=\mathrm{T}, \alpha_{1}=\varepsilon$ and $\alpha_{\mathrm{i}}=(2: \mathrm{n})$ complementary variables that defined the considered system.

Combining the first and second principles of thermodynamics, being $e$ the internal energy and $s$ the variable entropy, $\psi$ function is so defined:

$\psi=e-T^{*} s ; \quad \psi=\psi\left(T, \varepsilon, \alpha_{i}\right) ; \quad s=-\frac{\partial \psi}{\partial T} ; \quad \frac{\partial \psi}{\partial \varepsilon}=\frac{\partial e}{\partial \varepsilon} ; \quad \frac{\partial \psi}{\partial \alpha_{i}}=\frac{\partial e}{\partial \alpha_{i}} ; \quad$ with $i=2: n$

The local equation of heat conduction can be written: 


$$
\rho C T-\nabla(K: \nabla T)=\underbrace{\left(\sigma-\rho \frac{\partial \psi}{\partial \varepsilon}\right): \varepsilon-\rho \frac{\partial \psi}{\partial \alpha}: \alpha}_{\mathrm{d}_{1}}+\underbrace{\rho T \frac{\partial^{2} \psi}{\partial T \partial \varepsilon}: \varepsilon}_{\mathrm{S}_{\mathrm{the}}}+\underbrace{\rho T \frac{\partial^{2} \psi}{\partial T \partial \alpha}: \alpha}_{\mathrm{S}_{\mathrm{ic}}}+r_{\text {ext }}
$$

where $\rho$ is the mass density, $C$ the specific heat, $K$ the material conduction tensor, $\sigma$ the Cauchy stress tensor and $\nabla(K: \nabla T)$ is the thermal flux for conduction in material.

$\mathrm{d} 1$ is the power portion that become heat in irreversible way; this phenomenon occurs in the presence of plastics, viscoplastics or fatigue damage processing.

$\mathrm{s}_{\text {the }}$ is the thermomechanical coupling sources consisting of the thermoelastic source, $s_{i c}$ represents the other internal coupling sources and $r_{\text {ext }}$ is the external volume heat supply.

The overall heat source can then be theoretically estimated by evaluating the left-hand side of heat equation. Nevertheless, the thermal information given by the IR camera only concerns surface temperature fields. The heat source evaluation throughout the specimen gauge part (3D inverse problem) is an ill-posed problem that is impossible to solve without any information on the heat source distribution. If thermal gradients remain small throughout the thickness of the specimen, a depthwise averaged heat source distribution can be still be estimated.

To perform the heat source evaluation, the following hypotheses were formulated:

1) the heat conduction is isotropic and the related coefficient $k$ remains constant during the test; 2) the density $q$ and the specific heat $C$ are material constants, independent of the internal state; 3 ) the convective terms included in the material time derivation are neglected; 4) the external heat supply $\mathrm{r}_{\mathrm{ext}}$ (here only due to radiation heat exchanges) is time independent. Consequently, the equilibrium temperature field $T_{0}$ verifies the equation:

$$
-k \Delta T=r_{e x t}
$$

The temperature variations $\mathrm{h}=\mathrm{T}-\mathrm{T}_{0}$ induced by fatigue mechanisms are too small to have any influence on the microstructural state. It is then possible to state that $\mathrm{s}_{\mathrm{ic}}=0$. Under these hypotheses, the local heat Eq. (1) can be rewritten in the following simplified form:

$$
\rho C \frac{\partial \theta}{\partial t}-K \Delta \theta=d_{1}+s_{\text {the }}=s
$$

where $s=d_{l}+s_{\text {the }}$ is henceforth the overall volume heat source s.

In order to assess $s_{\text {the }}$ and then obtaining $d_{1}$ Thermoelastic Stress Analysis (TSA) can be used. The classical theoretical approach for TSA, that is a full field experimental technique for the stress field measurement and requires no contact with specimen under investigation, is shown as follows.

Expressing the equation of energy conservation for an isotropic material, in the case of small changes in temperature and adiabatic conditions and in the case of one dimensional stress with load sine we have [6], [7]:

$$
\rho_{0} C_{\varepsilon} \frac{\partial T}{T_{0}}=-\left(\alpha-\frac{1}{E^{2}} \frac{\partial E}{\partial T} \sigma_{m}\right) \Delta \sigma \sin \omega t-\frac{1}{4 E^{2}} \frac{\partial E}{\partial T}(\Delta \sigma)^{2} \cos 2 \omega t
$$

where $\sigma_{\mathrm{m}}$ and $\sigma_{\mathrm{amp}}$ are the mean and amplitude of the applied stress respectively and $\omega$ is the loading frequency, $T$ is the thermodynamic temperature (Kelvin); $\rho_{o}$ is the density $\left(\mathrm{kg} / \mathrm{m}^{3}\right) ; C \varepsilon$ is the specific heat under constant strain $\left(\mathrm{N} \mathrm{m} /{ }^{\circ} \mathrm{C} \mathrm{kg}\right) ; \alpha$ is the coefficient of thermal expansion $\left({ }^{\circ} \mathrm{C}^{-1}\right) ; E$ is the Young's modulus $\left(\mathrm{N} / \mathrm{m}^{2}\right)$.

The equation above shows that the thermal response of a solid body subjected to a purely sinusoidal load is the algebraic sum of a first part with pulsation $\omega$ equal to the pulse of load, which is a function of both amplitude loading and the mean load, and a second part, with pulsation $2 \omega$. 
Neglecting the variations of Young's modulus E and Poisson's ratio $v$ with temperature and expressing the specific heat at constant strain in terms of specific heat with constant pressure we obtain the relation between the change temperature of a solid and the variation of the trace of stress tensor for a plane stress state under the added hypotheses of local adiabatic condition, solid homogeneous and isotropic loaded in its linear elastic field and small temperature changes:

$$
\Delta T=-K_{0} T\left(\Delta \sigma_{1}+\Delta \sigma_{2}\right)
$$

with $\mathrm{K}_{0}$ thermoelastic constant of the material expressed as $\mathrm{K}_{0}=\alpha / \rho^{*} \mathrm{C}_{\mathrm{p}}$ where $\mathrm{C}_{\mathrm{p}}$ is the specific heat at constant pressure.

Equation (6) is valid only under the hypotheses of local adiabatic conditions and linear elastic behaviour of material. In case of non adiabatic condition or in presence of plastic behaviour such as at the crack tip a phase shift occurs between thermographic signal and reference signal [9] provided i.e. by a load cell or a strain gage on the specimen. In the following paragraphs show how the phase shift represents a powerful tool for the evaluation of plastic zones and location of cracks.

\section{Experimentation}

All the test have been performed on a Schenck PC400m servohydraulic fatigue machine with $250 \mathrm{kN}$ capacity. The thermoelastic and thermography data have been acquired via the differential IR camera DeltaTherm 1560 (DT) made by StressPhotonics (USA) with thermal sensitivity (NEdT) $<18 \mathrm{mK}$ and based on a InSb photonic detector with $320 \times 256$ pixel.

The three specimens tested were manufactured from steel Fe 37 with dimensions and geometry shown in Figure (1). Before the tests, specimens were painted with matt black paint to make uniform the emissivity of the treated surfaces and avoid reflections due to heat sources placed near the specimens during the test.

Strain gauge for each side of the specimen (Figure 1) were used to assess bending out of plane due to geometry of the specimen after welding and eventual not perfect alignment of the loading grips.

A thermographic fatigue test on the welded joints were conducted according to literature $[1,2]$. Uniaxial tensile loading test was applied (stress ratio $\mathrm{R}$ of 0.1 and frequency of $18 \mathrm{~Hz}$ ) for 20.000 cycles and then the amplitude was increased as stated in Table 1 up to the failure of the joints. The test started with an amplitude stress level of $50 \mathrm{MPa}$.

The entire test was monitored with infrared thermocamera, providing, at regular time intervals, thermoelastic images and thermographic sequences.

For good measure of the specimen surface temperature and to avoid influences from environmental conditions, the specimen was enclosed in a wood chamber. To assess bending out of plane due to an imperfect alignment of clamps of the machine loading, a mirror was used to frame simultaneously with the camera, front and rear surfaces of the specimen as shown in Figure 2.

Strain gauges data on the front and rear of the specimen, show the absence of bending out of plane, confirmed also by mean values of thermoelastic signal measured on front and on the mirror image of the specimen.

\section{Experimental data e results}

The aim of this paper is to assess the damage in steel welded joints by means of thermography and thermoelastic data. In particular, the surface temperature and phase variation of the thermoelastic signal were analysed. 


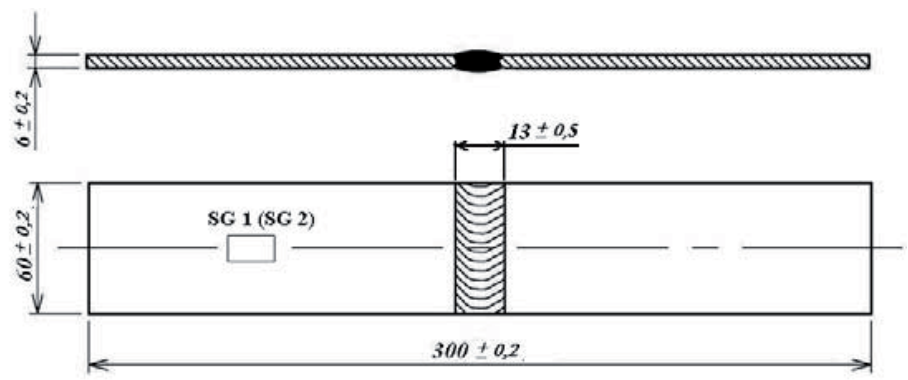

Fig 1. Dimensions and geometry of specimen and strain gauges locations

Table 1. Test performed on the specimens.

\begin{tabular}{|c|c|c|c|c|c|c|}
\hline \multirow{2}{*}{$\mathrm{N}^{\circ}$ step } & \multicolumn{2}{|c|}{ Specimen 1 } & \multicolumn{2}{c|}{ Specimen 2 } & \multicolumn{2}{c|}{ Specimen 3 } \\
\cline { 2 - 7 } & $\Delta \mathrm{F}[\mathrm{N}]$ & $\Delta \sigma$ nom & $\Delta \mathrm{F}$ & $\Delta \sigma$ nom & $\Delta \mathrm{F}$ & $\Delta \sigma$ nom \\
& & {$[\mathrm{MPa}]$} & & {$[\mathrm{MPa}]$} & & {$[\mathrm{MPa}]$} \\
\hline 1 & 9000 & 50 & 9000 & 50 & 9000 & 50 \\
\hline 2 & 14400 & 80 & 14400 & 80 & 14400 & 80 \\
\hline 3 & 19800 & 110 & 19800 & 110 & 19800 & 110 \\
\hline 4 & 25200 & 140 & 25200 & 140 & 25200 & 140 \\
\hline 5 & 30600 & 170 & 30600 & 170 & 30600 & 170 \\
\hline 6 & 33300 & 185 & 33300 & 185 & 33300 & 185 \\
\hline 7 & 36000 & 200 & 36000 & 200 & 36000 & 200 \\
\hline 8 & 38700 & 215 & 38700 & 215 & 38700 & 215 \\
\hline 9 & 41400 & 230 & 41400 & 230 & 41400 & 230 \\
\hline 10 & 44100 & 245 & 44100 & 245 & 44100 & 245 \\
\hline 11 & 46800 & 260 & 46800 & 260 & 46800 & 260 \\
\hline 12 & 49500 & 275 & 49500 & 275 & 49500 & 275 \\
\hline 13 & broken & broken & 52200 & 290 & 54900 & 290 \\
\hline 14 & broken & broken & 54900 & 305 & broken & broken \\
\hline
\end{tabular}

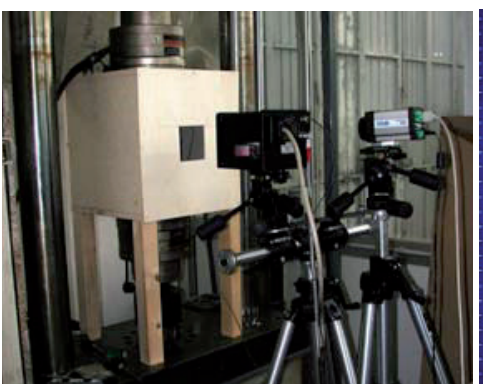

a)

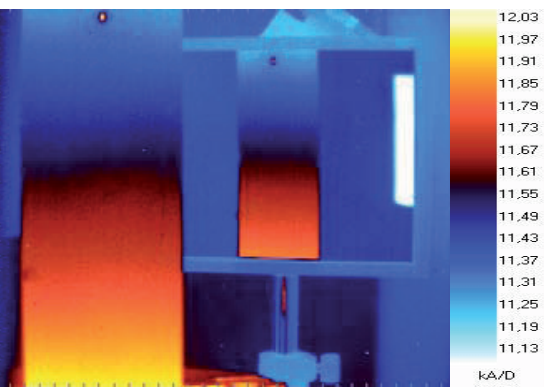

b)

Fig 2. a)Set-up and wood chamber used in the test b) and thermographic acquisition during the test. It is evident theheat flow due to the presence of hot oil in lower actuator

Thermoelastic and thermographic images were processed in Matlab ${ }^{\circledR}$ for improving the signal to noise ratio and for a better localization of damaged areas on the specimen. In fact, as it can be 
noticed from Figure 2, for example, the specimen during testing have been affected by a heat flow from the bottom to top due to the presence of hot oil in lower actuator.

We can monitor the damage growth during the entire test. Maximum temperature and phase were obtained by subtracting each image a reference image taken during the first load step when there was no damage. Monitoring for each step, characterised by a specific $\Delta \sigma$, an area around the welding on the front and rear of the specimen, the values of $\Delta T \max$ and $\Delta \Phi \max$ were obtained. In Figure 3 an example of the temperature and phase images are shown for specimen 1 , front view.

In the same figure can be noticed the method to obtain the fatigue limit $[1,2]$ usually adopted for base material, that was used in this work to characterise the welded joint fatigue behaviour.
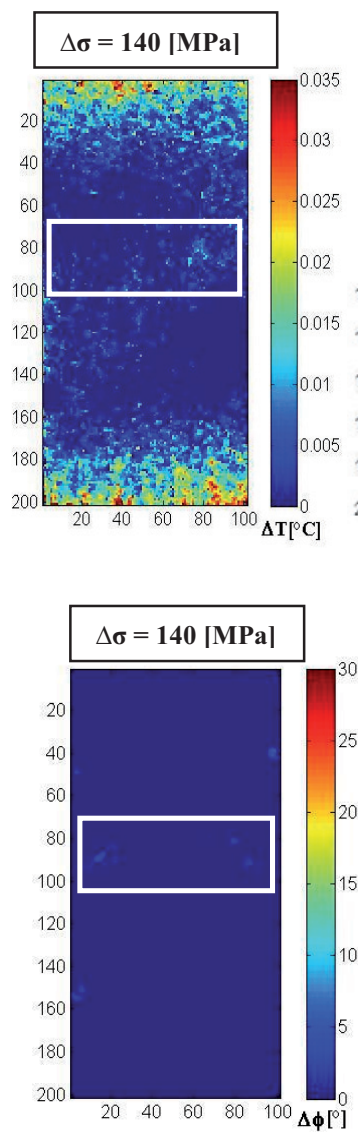
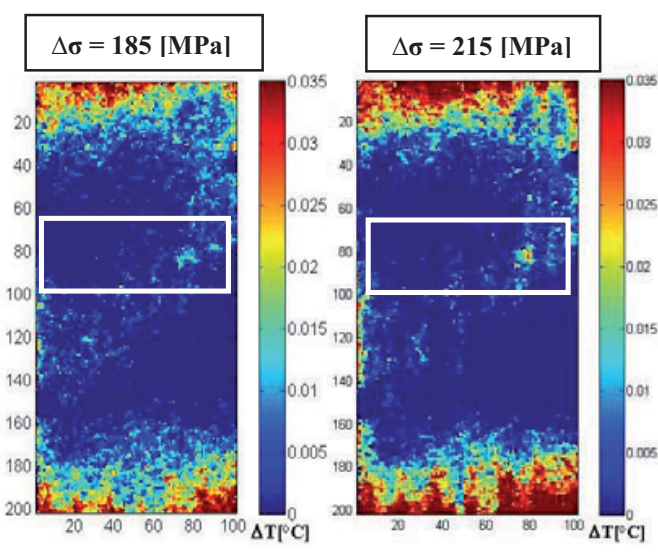

a)
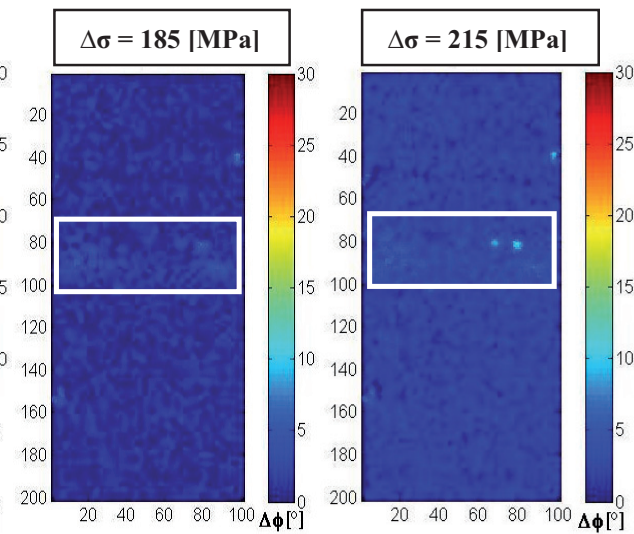
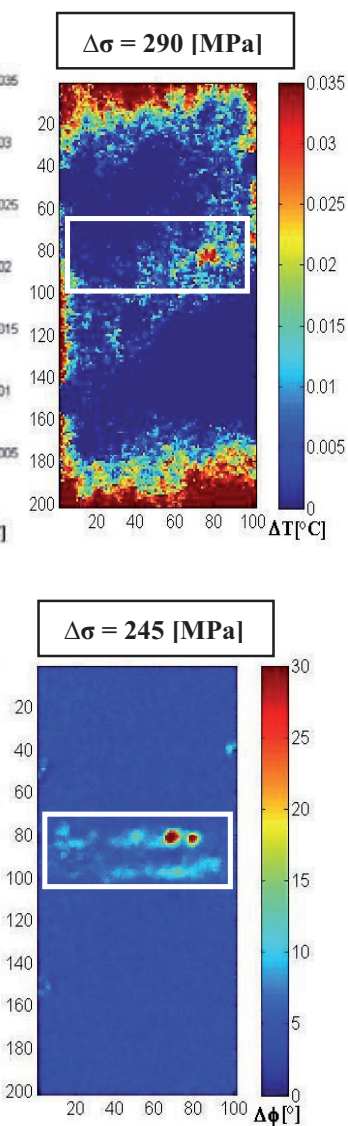

b)

Fig 3. Temperature a) and phase b) images for specimen 1, front view

Images acquired during the various steps loading show how is difficult to identify the damaged areas in the temperature image because temperature changes are very small. The temperature variations in welded joints are very low because the failure mechanisms and therefore the damage is different from the standard specimens that showing temperature variations much higher. Temperature changes of about a tenth of degree requiring a high performance equipment. The phase variations are much higher and allow to locate the damaged area more precisely than temperature. Variations in the value of signal phase indicate a not elastic behaviour and plastic dissipation in the material. The phase changes are due to high gradients of stress that may be ascribed to the non-adiabatic conditions and to the plastic behaviour of the crack tip. Patterson [5, 4] has shown that the performance characteristic of the phase signal at the crack tip contains a double reversal of sign, notably due to 
the two cited effects that have the opposite sign influence. Phase, thus, can be considered as an effective parameter for the identification of local damage.

These remarks show that the thermoelastic technique and more precisely the phase can be considered an effective parameter to evaluate the fatigue damage in materials. So in this paper proposes to use the phase change rather than temperature change for assessing the fatigue limit of materials with the methods in the literature.

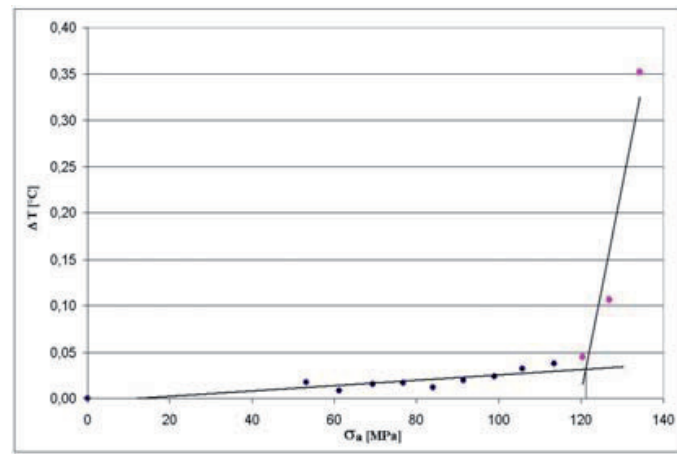

a)

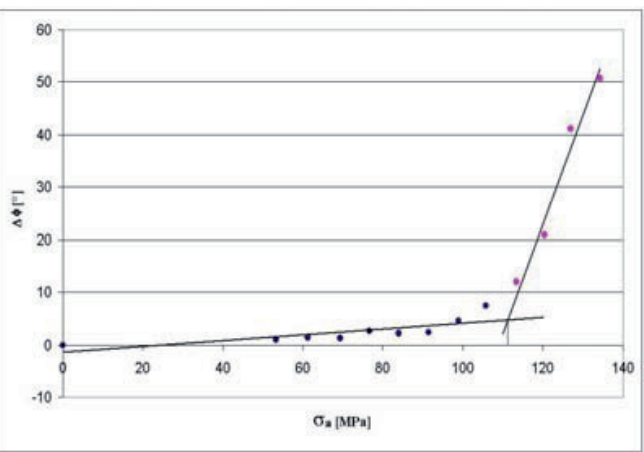

b)

Fig 4. a) Fatigue limit evaluation by means temperature and b) phase variation on specimen 1 , front view

Table 2. Fatigue limit evaluation on front and rear of the specimens.

\begin{tabular}{|c|c|c|c|c|}
\hline \multicolumn{4}{|c|}{ Fatigue Limit $\sigma_{\mathrm{L}}[\mathrm{MPa}]$} \\
\hline $\begin{array}{c}|c| \\
\text { Fatigue } \\
\text { parameter }\end{array}$ & \multicolumn{2}{|c|}{$\left.{ }^{\circ} \mathrm{C}\right]$} & \multicolumn{2}{c|}{$\Delta \varphi\left[^{\circ}\right]$} \\
\hline & front & rear & front & rear \\
\hline specimen 1 & 121 & 121 & 110 & 112 \\
\hline specimen 2 & 123 & 123 & 124 & 125 \\
\hline specimen 3 & 128 & 128 & 132 & 125 \\
\hline
\end{tabular}

In Figure 4 to obtain the fatigue limit two linear regression lines were used. Figure 4 and Table 2 show that the use of phase as parameter for assessment of the fatigue damage, gives results comparable to the results obtained using the temperature and consistent with data in literature that provide for this kind of joint a range between 90 to $140 \mathrm{MPa}$ for the fatigue limit.

Moreover it can be noticed that rear and front view shows substantially the same values.

Excellent agreement between the data obtained with consolidated thermographic technique and the new method based on the phase monitoring.

\section{Conclusions}

The aim of this paper is to present two novelties in the thermomechanical approach for fatigue rapid testing. A technique that is quite consolidated for base material, based on dissipated energy assessment in term of change of surface temperature of assess the damage in steel welded joints by means of thermography and thermoelastic stress analysis data. In particular, the variation of surface temperature and the phase of the thermoelastic signal were parameters used to assess the endurance limit of the joints.

Three Fe37 steel welded joints specimens are studied for assessment of fatigue damage. The classical fatigue parameter, change of temperature, and thermoelastic data, change of phase, are used to monitoring fatigue damage. In particular, after a filter processing on the thermographic and thermoelastic image, the analysis of damage areas has shown for temperature and phase variation, results in excellent agreement. 
Therefore, not only the consolidated thermographic techniques can be used to determine the fatigue limit of welded joint but also the phase information of thermoelastic signal can be used for the same reason with a much higher signal to noise ratio and much simpler data processing.

The proposed method can be very effectively be used for monitoring real welded structure since test showed that: the difference in monitoring front or rear surface of the welding is not significant; the phase change quite abruptly when the fatigue limit is exceeded and it is simple to monitor TSA phase each 20-30 seconds.

Furthermore TSA can be very useful for assessing the heat sources in classic thermomechanical approach providing information both of the stress field and of the area subjected to plastic deformation area (dissipation source) with phase information.

\section{References}

1. F. Curà, G. Curti, A. E. Gallinatti, XXVI Convegno Nazionale AIAS, (Torino, 2009)

2. A. E. Morabito, A. Chrysochoos, V. Dattoma, U. Galietti, International Journal of Fatigue, 29, 977-984 (2007)

3. G. La Rosa, A. Risitano, International Journal of Fatigue, 22, 65-73, (2000)

4. C. Casavola, U. Galietti, D. Modugno, C. Pappalettere, SPIE, (2006)

5. F.A Diaz., J.R. Jates, E.A. Patterson, International Journal of Fatigue, 365-376, (2004)

6. Wong A.K., J. Phys. Chem. Solids, 52, 483-494, (1991)

7. A.L. Gyekenyesi, G.Y. Baaklini, NASA/TM, (2001)

8. G. Curti, G. La Rosa, M. Orlando, A. Risitano, XIV Convegno Nazionale AIAS, (Catania, 1986)

9. G. Curti, A. Geraci, A. Risitano, ATA Ingegneria Automotoristica, 10, 634-636 (1989)

10. G. Fargione, A. Geraci, G. La Rosa, A. Risitano, M. Grench, XXVI Convegno Nazionale AIAS, (Catania, 1997)

11. G. Fargione, A. Geraci, G. La Rosa, A. Risitano, International Journal of Fatigue, 24, 11-19, (2002)

12. A. Blarasin, R. Fracchia, M. Pozzati, ATA, 51, (1998)

13. A. E. Morabito, V. Dattoma, U. Galietti, SPIE, (2002)

14. MP. Luong, Mech. Mater., 28, 155-63, (1998)

15. A. Chrysochoos, H. Louche, Int. J. Eng. Sci., 38, 1759-88, (2000)

16. T. Boulanger, A. Chrysochoos, C. Mabru, A. Galtier, International Journal of Fatigue, 28, (2004)

17. Wong A.K., Sparrow J.G., Dunn S.A., Journal of Physics and chemistry of solids, 49, 395-400, (1988).

18. S. Quinn, J.M.Dulieu-Barton and J.M. Langlands, Strain, 40, 127-133, (2004).

19. Dulieu-Smith S.M., Strain, 31, 9-16, (1995).

20. Dulieu-Barton J.M., Strain, 35, 35-39, (1999).

21. P. Stanley, W. K. Chan, Proceedings of International Conference of Fatigue of Engineering Materials and Structures, IMechE, (Sheffield (UK), 1986).

22. EUROCODE 3 - Design of steel structures. Part 1: general rules and rules for buildings. Final draft, (Bruxelles, 1992).

23. V. Dattoma, C. Pappalettere, J. of Strain Analysis, 36, 605-610, (2001) 